\title{
Low energy tertiary beam line design for the CERN neutrino platform project
}

\author{
N. Charitonidis* and I. Efthymiopoulos \\ CERN, 1211 Geneva 23, Switzerland \\ (Received 2 March 2017; published 7 November 2017)
}

\begin{abstract}
In the framework of CERN neutrino platform project at the CERN SPS North Area, two new beam lines have been designed as extensions of the existing secondary beam lines, able to provide low energy particles in the momentum range of 0.4 to $12 \mathrm{GeV} / c$. The layout of these very low energy beam lines, their design parameters as well as outlook on their expected performance, are summarized in this paper.
\end{abstract}

DOI: 10.1103/PhysRevAccelBeams.20.111001

\section{INTRODUCTION}

The CERN's North Area Experimental Halls (EHN1, EHN2, and ECN3) are multipurpose facilities able to host large fixed-target experiments as well as test beam stands. They are served by a network of beam lines, designed to deliver a wide spectrum of secondary and tertiary particle beams of varying and flexible composition (hadrons and leptons) as well as primary ion and attenuated proton beams. The momentum spectrum available in the beam lines extends from 10 up to $400 \mathrm{GeV} / c$. The low energy limit in the lines is defined mainly by the particle production flux and hardware limitations in the main bending magnets, while the upper limit corresponds to the maximum Super Proton Synchrotron (SPS) momentum extracted in the facility. The EHN1 experimental hall described in [1] is a general purpose industrial building, hosting four secondary beam lines named $\mathrm{H} 2, \mathrm{H} 4, \mathrm{H} 6$ and $\mathrm{H} 8$. These secondary particle beams are produced by the interaction of the primary proton beam with a momentum of $400 \mathrm{GeV} / c$ slowly extracted from SPS, on two thin beryllium plate primary targets, named $\mathrm{T} 2$ and $\mathrm{T} 4$, located $\sim 15 \mathrm{~m}$ underground to optimize the radiation shielding. The $\mathrm{H} 2$ and $\mathrm{H} 4$ beam lines, of interest here, emerge from the T2 target and are approximately $600 \mathrm{~m}$ in length, with the last $\sim 250 \mathrm{~m}$ inside the EHN1 experimental hall.

To host the CERN neutrino platform [2] experiments and tests, the EHN1 hall is extended by approximately $70 \mathrm{~m}$ in its length. Consequently, the $\mathrm{H} 2$ and $\mathrm{H} 4$ beam lines are extended in tertiary mode (i.e. beam branches emerging

\footnotetext{
Corresponding author. nikolaos.charitonidis@cern.ch

Published by the American Physical Society under the terms of the Creative Commons Attribution 4.0 International license. Further distribution of this work must maintain attribution to the author(s) and the published article's title, journal citation, and DOI.
}

from an intermediate-secondary target) whose design is described in detail in this paper. The layout of EHN1 hall as well as its extension is shown in Fig. 1.

The first experiments proposed in the framework of the CERN neutrino platform are two large-size detector prototypes based on Liquid Argon Time Projection Chamber (LAr TPC) technology pioneered in the ICARUS GrandSasso detector [3]. They are named "ProtoDUNE-Dual Phase" (NP-02) and "ProtoDUNE-Single Phase" (NP-04), intended to test and validate technology options for the far detectors of the future DUNE facility under construction in the USA [4]. The required beam parameters for these experiments described in their scientific proposals $[5,6]$ are summarized in Table I.

The layout of the EHN1 extension and a view of the $\mathrm{H} 2$ and $\mathrm{H} 4$ beam line extensions reaching the detectors can be seen in Fig. 2.

The need of a special design for the $\mathrm{H} 2$ and $\mathrm{H} 4$ beam line extensions arises from the fact that the existing beam lines cannot provide the stable beams of controllable spectrum and particle content of the low range of momenta required by the experiments, and particularly the desired hadron beams. This is because their $\sim 600 \mathrm{~m}$ of length as mentioned above, prevents from transporting, the anyhow limited in number low energy pions and kaons produced at the primary target. They will decay before reaching the experiments making their use inefficient. In addition, to transport low energy particles below $10 \mathrm{GeV} / c$, the required current in the spectrometer magnets of the beam lines needs to be in the order of magnitude of a few Amperes, reaching that way the limit in terms of stability and precision of the power supplies, originally designed to operate close to the top SPS energy of $450 \mathrm{GeV} / c$.

\section{BEAM LINE DESIGN}

\section{A. Layout and parameters}

To overcome the aforementioned challenges for the requested hadron beams, the $\mathrm{H} 2$ and $\mathrm{H} 4$ beam lines are 


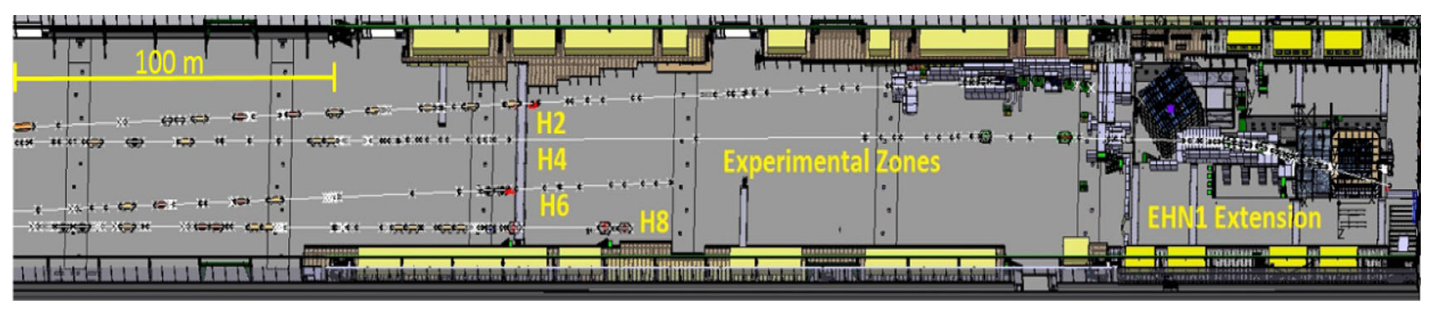

FIG. 1. 3D drawing of the EHN1 hall. On the left the upstream part of the four beam lines (H2, H4, H6 and H8) in the hall can be seen. The new extension part for the hall is shown on the right part.

extended in tertiary branches. The adopted conceptual design that satisfies the requirements of the experiments includes: a medium-energy secondary beam produced at the primary $\mathrm{T} 2$ target is transported to impinge on a secondary target from which the wanted low energy secondary particles are produced. These particles, following a momentum selection, are transported over the shortest possible distance to the neutrino platform experiments. By adjusting the intensity and the particle type of the secondary beam (mixed hadron or electron) as well as the secondary target material, a wide range of tertiary particle beams can be produced covering well the required spectrum, flux and particle type of the experiments. Tertiary low energy branches of the EHN1 beams were constructed in the past for the ATLAS and CMS experiments, to test the response of their detector prototypes, in particular calorimeters. Their performance was very satisfactory, although with less stringent requirements compared to the case discussed here $[7,8]$.

To produce the hadron low energy beams, the chosen secondary beam momentum is $80 \mathrm{GeV} / c$ which corresponds to the peak of the measured production rates of pions at the T2 target from the impinging $400 \mathrm{GeV} / c$ primary protons on beryllium [9]. The secondary beams can be operated in the range of $10^{6}$ particles per SPS spill (typically $4.8 \mathrm{~s}$ long) which as will be seen later is sufficient to generate the requested flux range for the tertiary beam. The tertiary branches downstream of the secondary target consist mainly of a momentum selection station with large dipoles, preceded by an acceptance stage and followed by a final focusing stage both made of large-aperture (200 $\mathrm{mm}$ diameter) quadrupole elements.

TABLE I. The main beam design parameters as described in the experimental proposals, of ProtoDune-DP (NP-02) and ProtoDune-SP (NP-04).

\begin{tabular}{|c|c|c|}
\hline Parameter & $\begin{array}{c}\text { ProtoDUNE_-DP } \\
\text { (NP-02) }\end{array}$ & $\begin{array}{c}\text { ProtoDUNE-SP } \\
(\mathrm{NP}-04)\end{array}$ \\
\hline Particle type & \multicolumn{2}{|c|}{$\pi^{+-}, \mu^{+-}, \mathrm{e}^{+-}, \mathrm{K}, \mathrm{p}$} \\
\hline Momentum & $<12 \mathrm{GeV} / c$ & $<7 \mathrm{GeV} / c$ \\
\hline$\Delta \mathrm{p} / \mathrm{p}$ & \multicolumn{2}{|c|}{$<5 \%$} \\
\hline Beam size rms & \multicolumn{2}{|c|}{$\sim 10 \mathrm{~cm}$ at the entrance of the cryostats } \\
\hline Maximum, rate & $100 \mathrm{~Hz}$ & $100 \mathrm{~Hz}$ \\
\hline
\end{tabular}

The momentum selection dipoles define the geometry of the tertiary beam lines. Their bending connects the location of the secondary target placed along the existing $\mathrm{H} 2$ and $\mathrm{H} 4$ lines, and the wished entry point in the detectors.

As seen in Fig. 2, the detector prototypes are installed in the extension part of the EHN1 building, inside two large pits at $\sim 7.5 \mathrm{~m}$ (for $\mathrm{H} 2$ ) and $\sim 9 \mathrm{~m}$ (for $\mathrm{H} 4$ ) below the floor of the existing hall. This construction, besides to optimize the installation of the detectors and resolve safety issues by the use of liquid argon, protects the detectors, being slow-readout devices-TPCs, from being fully exposed to the background accompanying the $\mathrm{H} 2$ and $\mathrm{H} 4$ beam lines. In addition, to maintain the overall budget at a reasonable ceiling, the use of available magnets and power supplies was preferred. In the final design, the bending dipoles are tilted by 35 or 60 degrees with respect to the building floor for $\mathrm{H} 2$ and $\mathrm{H} 4$ respectively, to provide a simultaneous deflection in both the horizontal and the vertical plane. The tilt of the bending dipoles, although it introduces additional technical challenges for the support and alignment of these 30-T objects, is preferred as it minimizes the length of the tertiary beam line, thus reduces flux losses due to decays. At the same time also allows using the full aperture of the dipoles $(420 \times 140 \mathrm{~mm})$ thus maintaining the particle flux that would otherwise be compromised by successive use of horizontal and vertical bends without additional elements in between to shape the beam. For the $\mathrm{H} 2$ beam line, four dipoles are used to provide a total deflection angle of $231.6 \mathrm{mrad}$, while for $\mathrm{H} 4$ two dipoles are used for the total deflection angle of $240.06 \mathrm{mrad}$, but at lower top energy. For the case of $\mathrm{H} 4$, an additional dipole after the final focusing stage is installed, aimed to allow deflecting the beam to reach additional entry points in the detector.

For the acceptance stage, downstream of the secondary target, three quadrupoles are used. They are arranged in the Defocus-Focus-Defocus (DFD) configuration and placed such to maximize the beam acceptance and minimize the overall length. For the final focusing, a quadrupole doublet is used, again designed to minimize the beam length matching at the same time the required beam spot parameters at the 


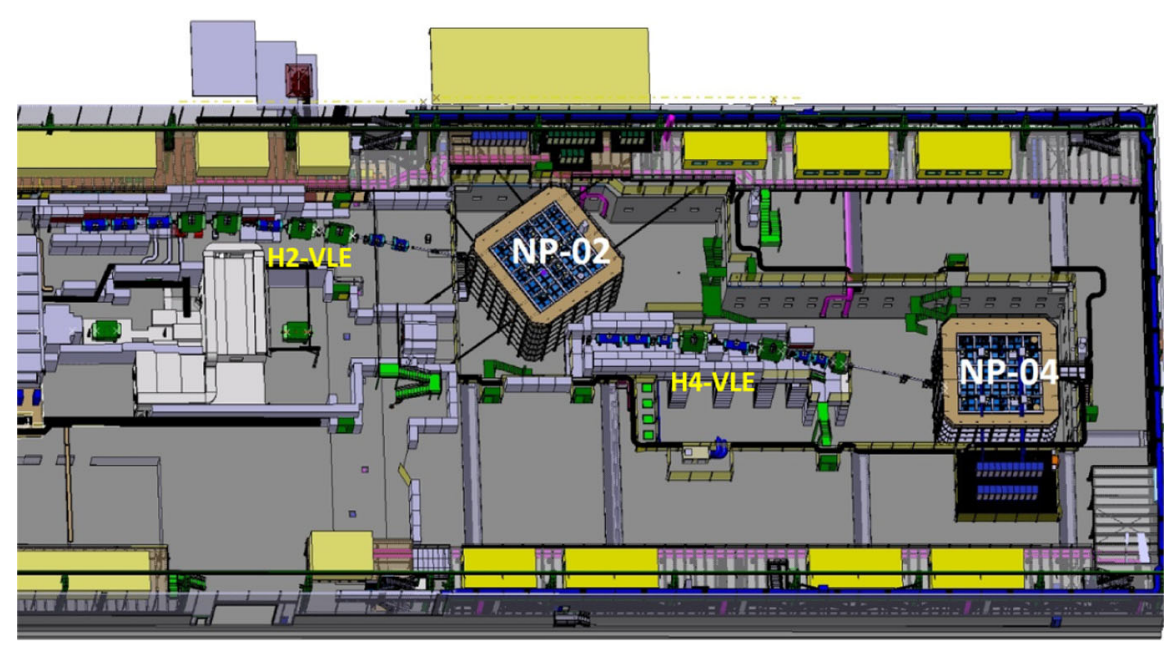

FIG. 2. 3D drawing showing the layout of the EHN1 hall extension housing the two detector prototypes for the NP-02 and NP-04 experiments. The $\mathrm{H} 2$ and $\mathrm{H} 4$ beam line extensions reaching the two experiments respectively are also visible.

experiment. Finally, a "field lens" quadrupole is used, located in the middle of the bending dipoles to allow for dispersion control and the momentum recombination. The beam design completes with a two-jaw collimator to allow for finer momentum bite selection, and the adequate beam instrumentation for particle trigger and tagging. All beam elements are titled to the bending plane defined by the dipoles, in order to minimize aperture loss and coupling of the horizontal and vertical planes in the optics. The beam line is under vacuum $\left(10^{-3}\right.$ mbar) over its full length. Concrete and iron shielding elements are placed around the secondary target in order to minimize the resulting radiation field and protect the surrounding counting rooms. A beam dump consisting of iron and concrete blocks, placed near the second dipole magnet, absorbs the high energy particles that have not interacted with the secondary target.

The two beam line extensions are designated H2-VLE and H4-VLE, the acronyms standing for very low energy to emphasize the reach in the $\mathrm{GeV}$ range of these beam lines. A schematic layout of the existing $\mathrm{H} 2$ beam line along with the newly designed extension can be seen in Fig. 3 for illustration. A conceptual drawing of the two VLE beam extensions is shown in Fig. 4, with an evident similarity between the two designs.

The instrumentation for the beam monitoring as well as the particle tagging is shown in Fig. 4, and explained in detail in Ref. [10].

Schematic 3D views depicting the new beam lines are shown in Figs. 5 and 6. The total length of the H2-VLE beam line starting from the secondary target up to the entrance of the NP-02 cryostat is $39.703 \mathrm{~m}$ while for H4VLE from the secondary target to the default entry of the NP-04 cryostat is $35.910 \mathrm{~m}$.

\section{B. Optics design}

The key element in the optics design of the VLE beams is the magnetic spectrometer formed by the large dipole magnets configured in a double bend achromat lattice. The spectrometer selects particles produced at the secondary target with an overall momentum acceptance of approximately $\pm 5 \%$. For the optics calculations, the TRANSPORT code [11] is used, which has been widely employed for the design of the particle beams at CERN. The resulting optics diagrams are shown in Figs. 7 and 8. The three lines in the

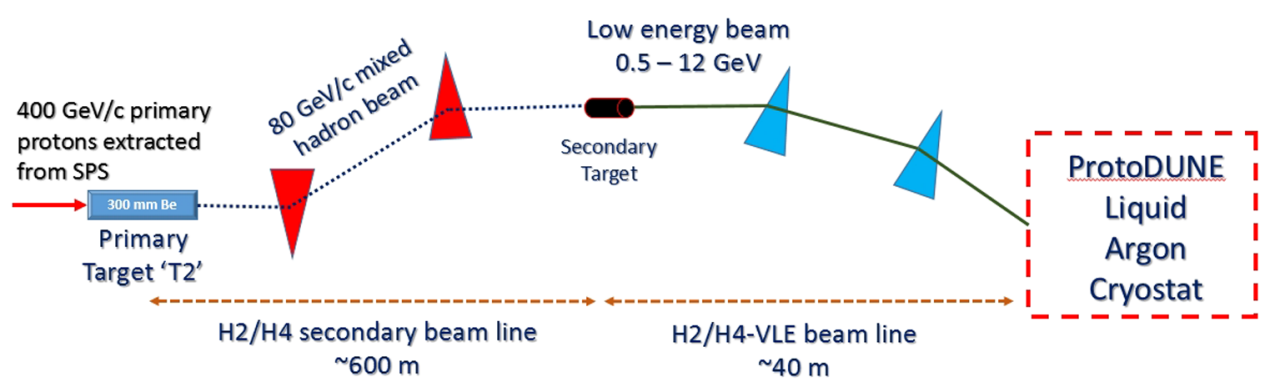

FIG. 3. Conceptual layout of the H2/H4 beam line and the newly designed VLE part, leading to the $11 \times 11 \mathrm{~m} \mathrm{NP-02/04} \mathrm{cryostat.}$ 

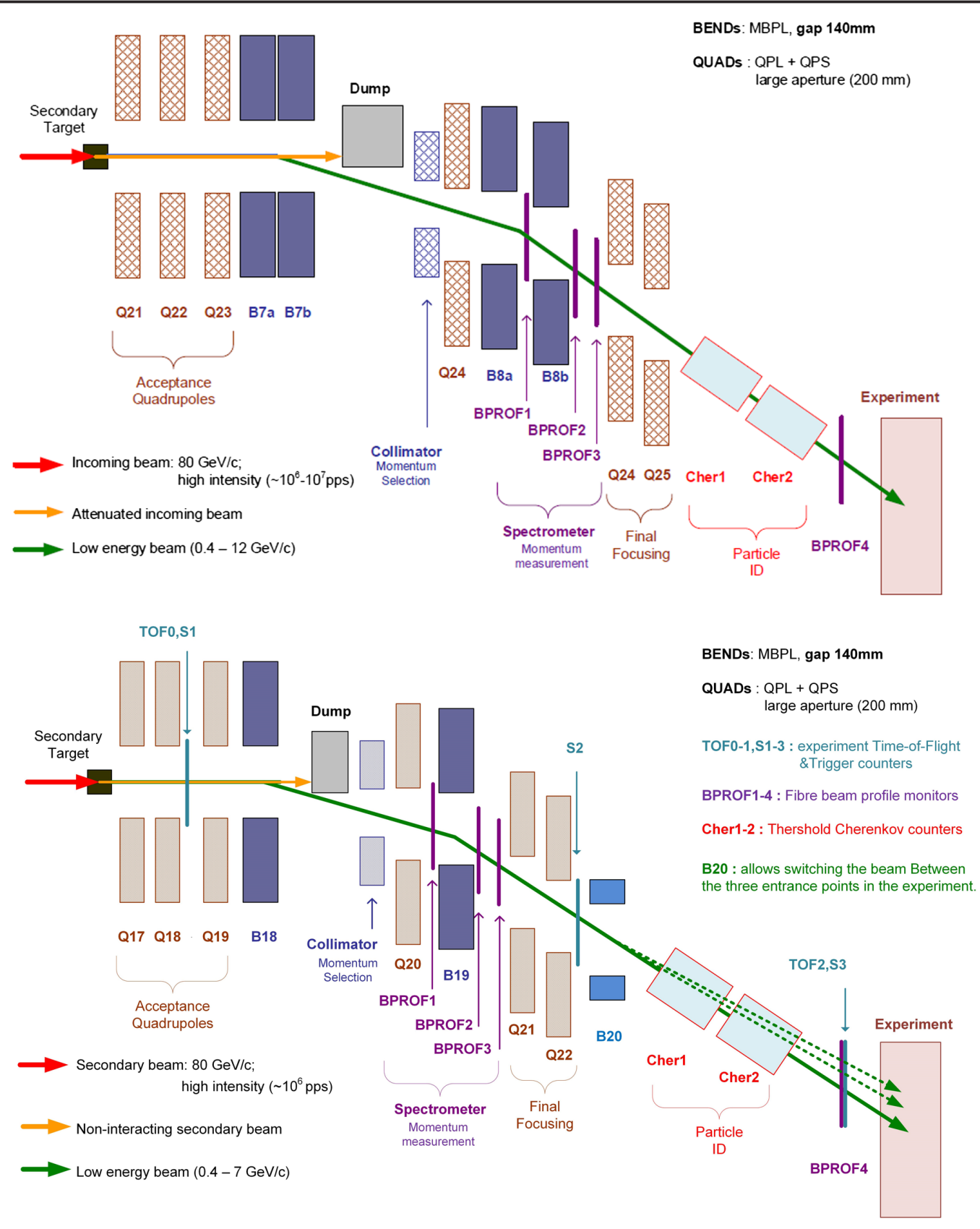

FIG. 4. Schematic layout of the H2-VLE (top) and H4-VLE (bottom) beam lines, showing the magnetic elements and the proposed beam instrumentation.

figures correspond to the trace of a particle emerging at an angle (sinelike ray-red line), or position (magnification term-green line) or at a different momentum (dispersion term-blue line) from the secondary target. At each location, the beam envelop is to be considered as the sum of the displacements in the three lines, each one contributing in different weight. This can be observed in the final focus position of the lines to the experiments, that is varying: for H2-VLE, the focal point is at the center of the experiment (position NP-02 middle in Fig. 7) while for H4VLE the focal point is at the entry point on their cryostat (position NP-04 Exoskeleton in Fig. 8). During data taking it is easy to rematch the optics of the lines to move the focal position or the beam waist along the line.

Both beam lines have been optimized to accept a maximum particle divergence from the pointlike target equal to $\pm 10 \mathrm{mrad}$. The nominal beam momentum is brought at focus in the middle of the spectrometer, where the dispersion term is maximized allowing a momentum analysis with a collimator having the jaws parallel to the beam bending plane. The field-lens quadrupole located next to the collimator recombines the off-momentum 


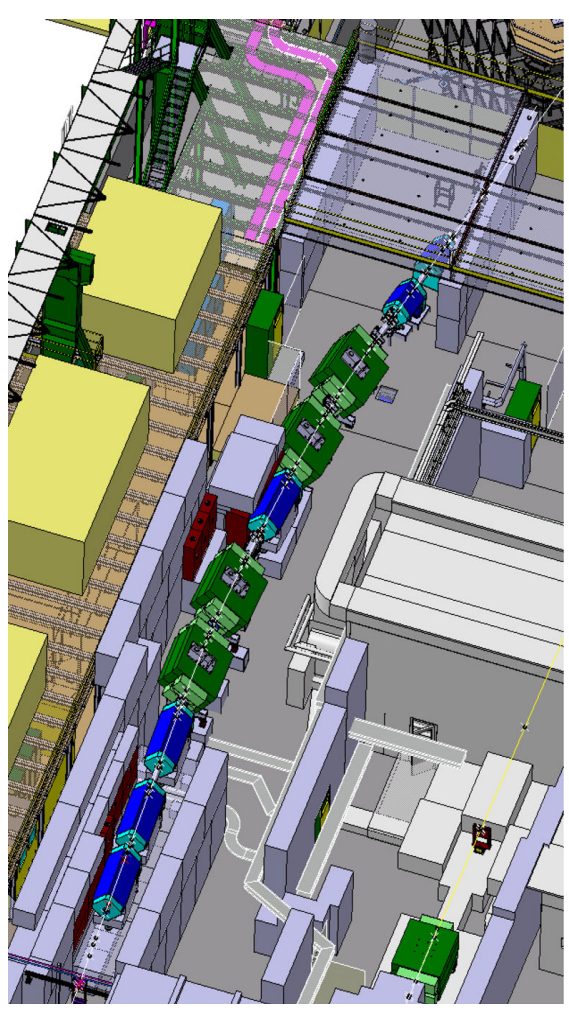

FIG. 5. 3D model layout of the H2-VLE beam line. The quadrupole triplet (in blue) and the target in front of it, are shown in the left of the picture. Part of the NP-02 cryostat is shown in the right of the picture. (Courtesy: V. Clerc and S. Girod)

particles at the exit of the spectrometer before the final focusing to the experiment with the last quadrupole doublet. Various space and position constraints between the elements have been imposed for example: the placement of vacuum equipment (flanges, bellows, pumping ports), space reservation for the instrumentation (from the beam monitoring but also from the experiments for particle trigger and tagging), and location of shielding blocks

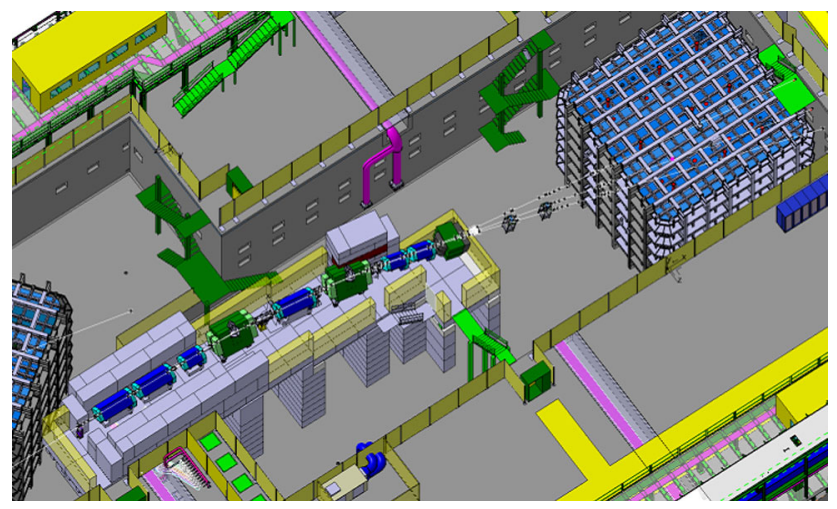

FIG. 6. 3D model layout of the H4-VLE beam line. The NP-04 cryostat is shown in the back. The tertiary beam goes from left to right and starts at the secondary target shown at the start of the concrete platform. (Courtesy: V. Clerc and S. Girod) allowing the service access to all elements in safe conditions. The requirement to maintain a minimal total length of the beam line, and the constraint to use existing magnets and power supplies to reduce the overall cost, lead to a nonzero dispersion derivative in the recombination position and therefore to a dispersion contribution at the bending plane to the beam spot size at the experiment. However, even for the full momentum acceptance of 5\%, this results in an approximate $0.5 \mathrm{~mm}$ contribution to the $\mathrm{H} 2$ beam spot size rms at the position of the experiment, with similar figures for $\mathrm{H} 4$, that is fully acceptable.

\section{Secondary beam line configuration and secondary target}

The new H2-VLE and H4-VLE beam extensions, being in fact prolongations of the existing lines downstream of the secondary targets, have no impact on the operation of the upstream beam lines (hosting numerous small and a few permanent experiments) throughout the year. As all other experiments, the neutrino platform ones would have to request a data-taking period during which the upstream beam lines would be configured to optimally produce the required particles from the secondary target. For the H2VLE beam all new equipment is located downstream of the end beam dump of today's configuration. For the H4 beam line, an additional horizontal dipole magnet is installed towards the end of the existing line. This dipole, powered in series with an existing one further upstream, would provide the necessary lateral deviation of the $\mathrm{H} 4$ line to avoid the large cryostat of NP-02 before hitting the secondary target of H4-VLE.

The optics of the existing $\mathrm{H} 2$ and $\mathrm{H} 4$ beam lines have been matched to provide a focal point at the location of the new secondary targets. The expected beam profiles of the secondary beam in the horizontal and vertical planes at the secondary target position are shown in Figs. 9(a) and 9(b), calculated using G4BeamLine ${ }^{\circledR}$, GEANT4 based simulation package [12] and the matched optics.

The secondary target is typically a solid rod, whose length and radius and material have been optimized to satisfy the experimental requirements for the particle rate and composition, as described in detail in Refs. [10,13] and summarized in Table II below. More specifically, for hadron beams, a copper target of $30 \mathrm{~mm}$ radius and $30 \mathrm{~cm}$ length (for momenta in the range of [4-12] $\mathrm{GeV} / c$ ) and a solid tungsten target (for momenta in the range of [0.4-3] GeV/c) have been chosen for H2-VLE. For the $\mathrm{H} 4-\mathrm{VLE}$ case, a larger rod of $50 \mathrm{~mm}$ radius of the same materials has been proposed. For electron beams target rods made of $\mathrm{Pb}$ of a few radiation lengths are proposed. The hardware of the secondary target installation allows multiple rods to be loaded in a mechanical support that can be changed remotely during operations, thus giving maximum flexibility to change between different particle types and energy range. 

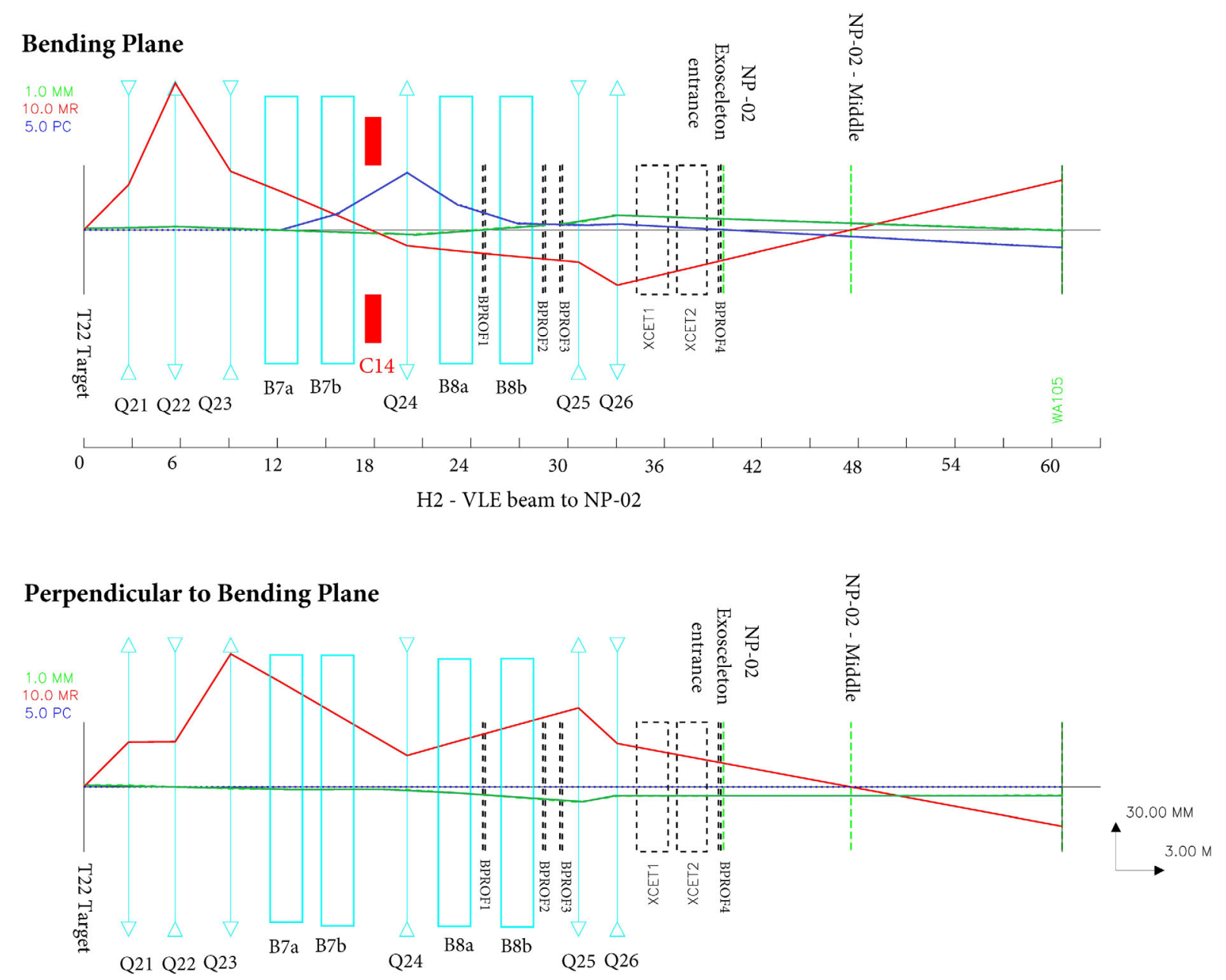

FIG. 7. Optics diagram for the H2-VLE beam line. The red line corresponds to the sinelike ray (particles emerging at 10 mrad from the target), the blue line represents the dispersive ray (at 5\%) and the green line the magnification term (corresponding to $1 \mathrm{~mm}$ at the origin).

\section{BEAM LINE PERFORMANCE}

The VLE beam line performance was extensively studied using Monte Carlo simulations. The beam line with all of its components-secondary target, magnetic elements, beam instrumentation as well as relevant parts of its surrounding shielding and structures-were modeled in detail using the G4BeamLine package. As an example, the model of the H2-VLE is shown in Fig. 10. In order to address the key issue in the simulation studies of hadron beams, namely the production of the low-energy particles in the secondary target, the two-main physics lists from GEANT4 interfaced in the G4BeamLine package were used: QGSP_BIC and FTFP_BERT. From the available literature, we understood the two physics lists depend on different parametrizations of the various cross sections, with no clear preference among them, resulting in variations on the predicted production flux of particles as a function of the momentum and production angle, something we also observed. A detailed benchmarking study of the simulation results with the test-beam data once the VLE beams become operational is being considered, although such a study has its own challenges and not trivial to realize. The transport threshold of particles was set to $10 \mathrm{MeV}$, taking into account all the physics processes included in GEANT4.

The main focus of the performance studies concerns the challenging case of hadron beams, affected by the production rates and particle decays. Detailed analysis, discussion on the results from the simulation studies concerning the VLE beam composition as well as the simulated trigger rate of the experiments is presented in Ref. [10]. Highlights of the key findings demonstrating the VLE beam performance for hadron beams is presented in the following paragraphs. The special case of electron or muon beams is described in the last section.

\section{A. Beam composition}

The H2-VLE beam composition within the vacuum beam pipe aperture at the entry of the NP02 cryostat for 

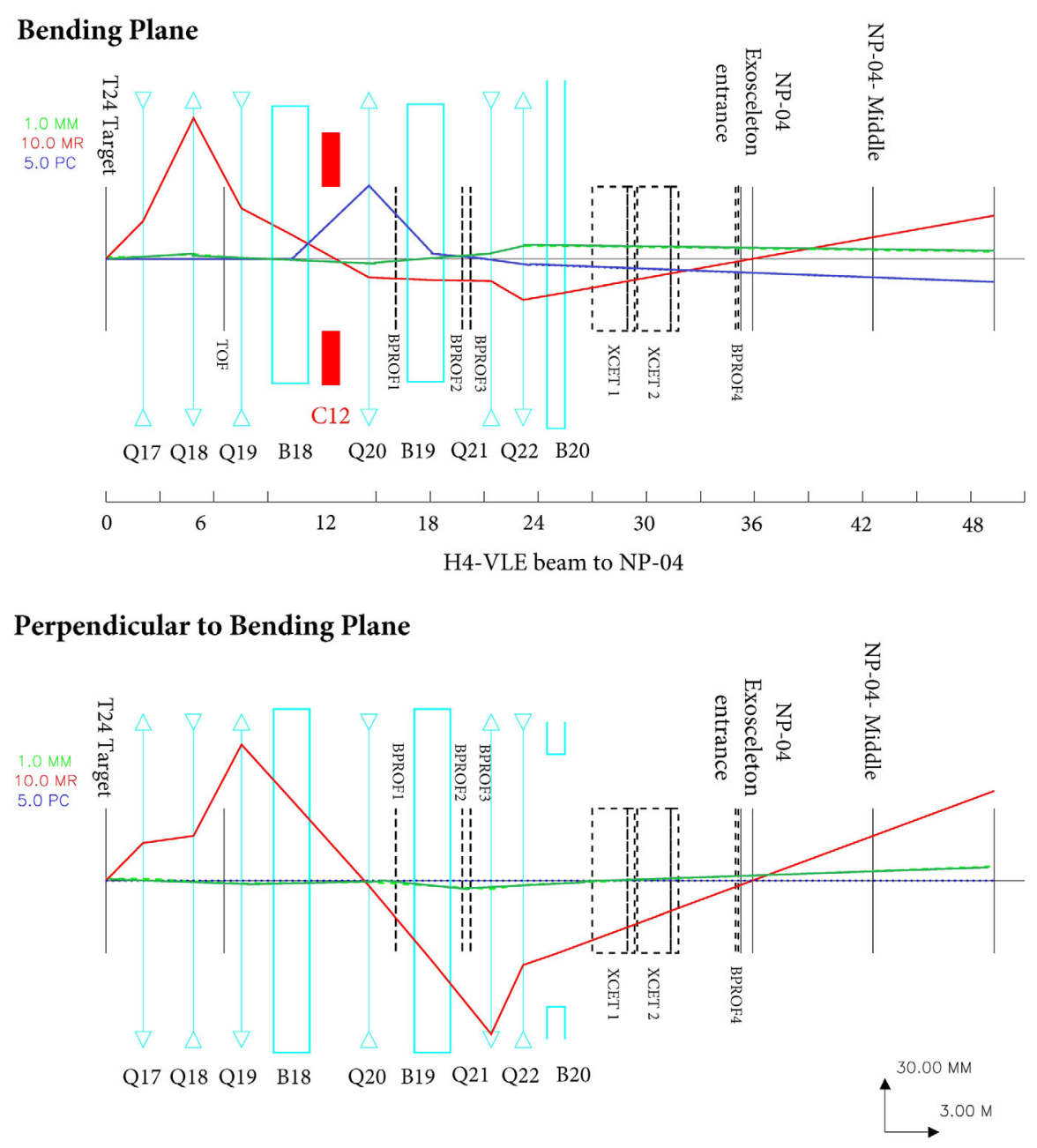

FIG. 8. Similar to Fig. 7 for the case of the H4-VLE line.

both positive and negative particle beams can be seen in Fig. 11, using the FTFP_BERT physics list. In this study, $10^{6}$ pions of $80 \mathrm{GeV} / \mathrm{c}$ have been simulated impinging on the secondary target, resulting to a statistical error in all cases of less than $3 \%$.

It is evident from Fig. 11 that in the case of hadron beams, the electrons and positrons are dominating the composition of the VLE beam in the lower part of the spectrum, in both the negative and positive configurations. At higher momenta, the pions constitute the majority of particles while a constant number of muons (mainly from pion decays) is always present at all momenta greater than $2 \mathrm{GeV} / c$.

For the trigger rate studies, the secondary particles were generated at the $\mathrm{T} 2$ target in order to include flux loses in the $\mathrm{H} 2$ line. Table III shows the resulting trigger rate for NP-02 experiment, assuming full acceptance in the $\mathrm{H} 2$ beam line transport, normalized to $10^{6}$ pions of $80 \mathrm{GeV} / \mathrm{c}$ produced at the T2 target. For the VLE part, a "good" trigger particle is one that traverses all detectors installed in the beam line shown in Fig. 3. As expected, the H4-VLE beam has similar performance, shown in Table IV, and described in detail in Ref. [7]. In the study, the combination of secondary target heads as described in Table II are used.

\section{B. Momentum selection}

The design of the VLE beams is based on the momentum spectrometer formed by the large dipoles. In both beam lines, the deflecting dipoles before and after the collimator are powered separately. The first dipole (or set of two for the case of H2-VLE) is used to trim the beam position, i.e. bring its center to the center of the collimator, compensating for various errors. The second dipole (or set of dipoles) is then used as the reference momentum selection spectrometer between the collimator center and the trigger counter of the experiment located downstream. The current in the spectrometer dipole is changed proportionally to assure the momentum definition linearity of the beam.

As explained above, based on the magnet apertures the full momentum acceptance of the beam is $\pm 5 \%$. For most of the cases, this momentum bite, uniformly distributed around the central value defined by the chosen VLE 
x-position @ T22

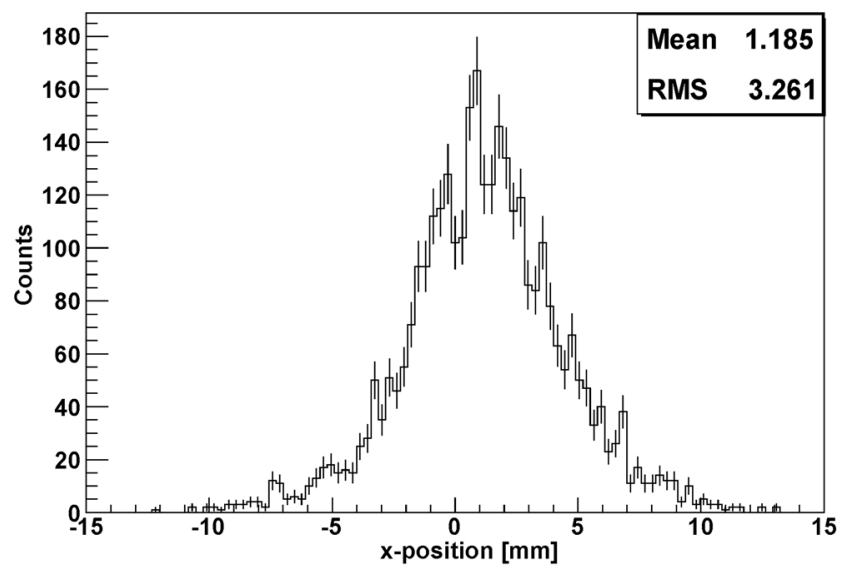

x-position @ T24

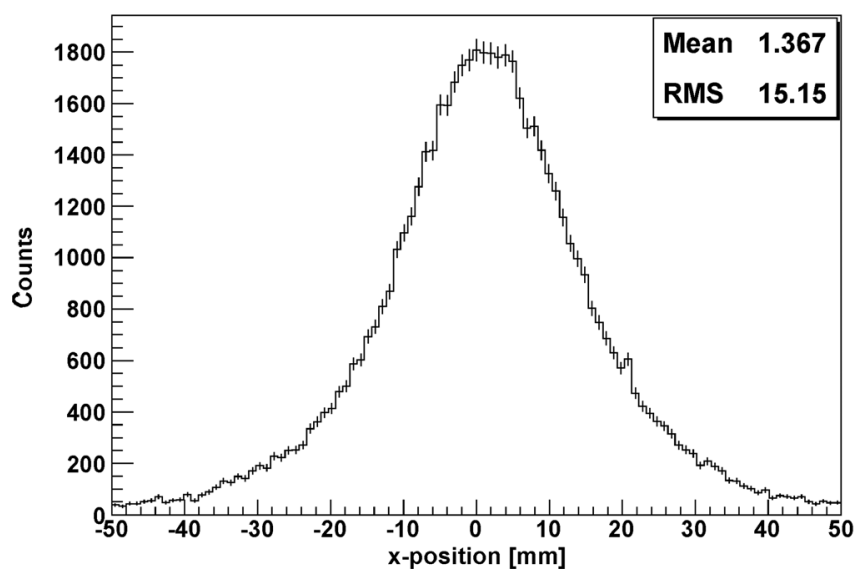

(c) y-position @ T22

(b)

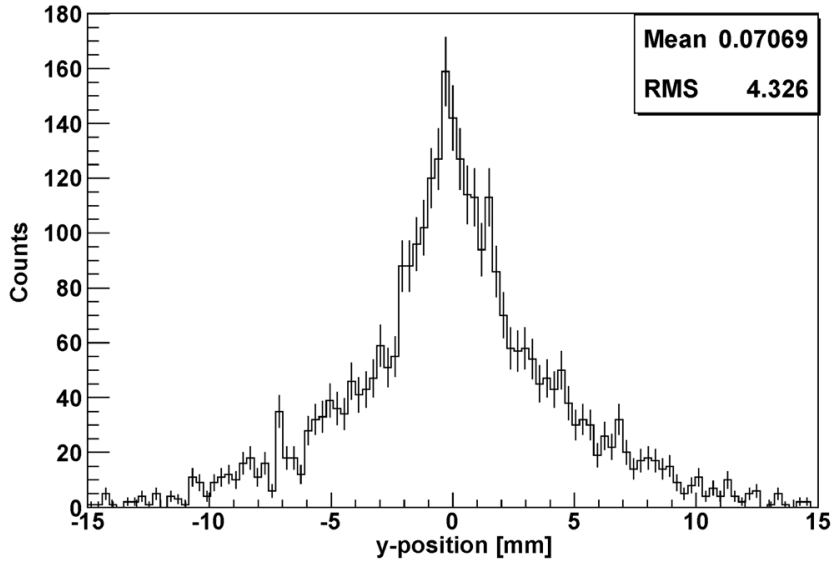

y-position @ T24

(d)

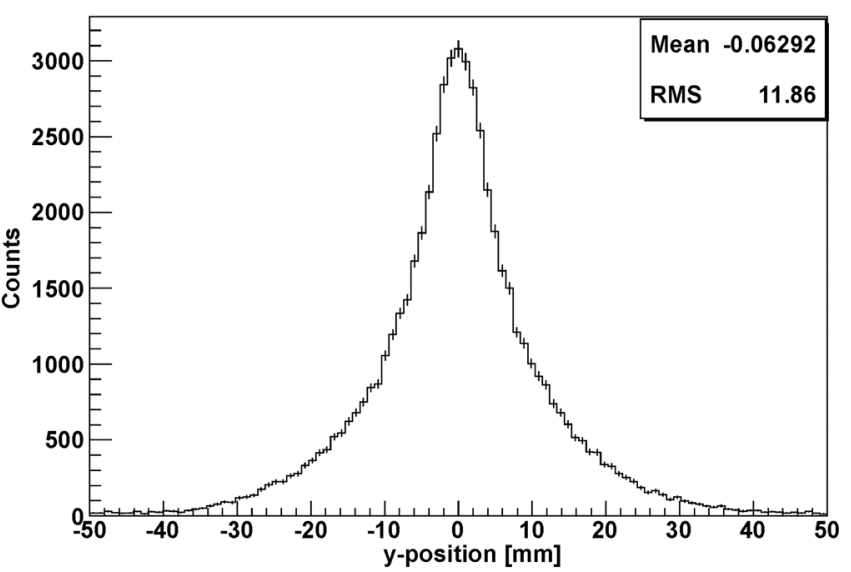

FIG. 9. (a) and (b) The H2 secondary beam profiles at the secondary target, in the horizontal (left) and the vertical (right) planes. (c) and (d) The H4 secondary beam profiles at the secondary target, in the horizontal (left) and the vertical (right) planes.

momentum, is sufficient for the experiments. However, using the installed collimator, the momentum spread in the beam can be reduced, down to $\pm 1 \%$ for an opening of $\pm 5 \mathrm{~mm}$ in the collimator jaws, of course with a penalty in the particle flux. The simulated effect of the momentum

TABLE II. Parameters of the secondary targets.

\begin{tabular}{|c|c|c|}
\hline Beam & Materia & Dimensions \\
\hline \multicolumn{3}{|l|}{ Hadron beams } \\
\hline \multicolumn{3}{|l|}{ H2-VLE } \\
\hline$[0.4,3] \mathrm{GeV} / c$ & $\mathrm{~W}$ & Rod $30 \mathrm{~mm}$ diam, $30 \mathrm{~cm}$ length \\
\hline$[4,12] \mathrm{GeV} / c$ & $\mathrm{Cu}$ & Rod $30 \mathrm{~mm}$ diam, $30 \mathrm{~cm}$ length \\
\hline \multicolumn{3}{|l|}{ H4-VLE } \\
\hline$[0.4,3] \mathrm{GeV} / c$ & W & Rod $50 \mathrm{~mm}$ diam, $30 \mathrm{~cm}$ length \\
\hline$[4,7] \mathrm{GeV} / c$ & $\mathrm{Cu}$ & Rod $50 \mathrm{~mm}$ diam, $30 \mathrm{~cm}$ length \\
\hline \multicolumn{3}{|l|}{ Electron beams } \\
\hline \multicolumn{3}{|l|}{ H2-VLE } \\
\hline $\begin{array}{l}\quad[0.4,12] \mathrm{GeV} / c \\
\text { H4-VLE }\end{array}$ & $\mathrm{Pb}$ & Rod $30 \mathrm{~mm}$ diam, $15 \mathrm{~mm}$ length \\
\hline$[0.4,7] \mathrm{GeV} / c$ & $\mathrm{~Pb}$ & Rod $50 \mathrm{~mm}$ diam, $15 \mathrm{~mm}$ length \\
\hline
\end{tabular}

selection collimator in the H2-VLE beam is shown in Fig. 12, for the case of a $4 \mathrm{GeV} / c$ beam.

An alternative method to compensate the loss of particle flux while closing the momentum selection collimator jaws for improved momentum resolution, is to measure the momentum of each particle using a position tracking around the VLE line spectrometer, i.e. second dipole (or dipoles). The technique has been successfully applied in the past [7] with excellent results. The simulated reconstruction of particle-by-particle momentum for the case of H2-VLE and for a $7 \mathrm{GeV} / c$ beam is shown in Fig. 13, and for three different spatial resolutions of the tracking detectors.

\section{Electron or muon beams}

The above sections concentrate in the production of hadron beams, where the resulting VLE beam reaching the experiment is a mix of hadrons and electrons in relative ratios varying with the beam momentum. Alternatively, low energy electron or muon beams can be easily configured and represent much less of a challenge to achieve at the wanted trigger rate of the experiment of $100 \mathrm{~Hz}$. 


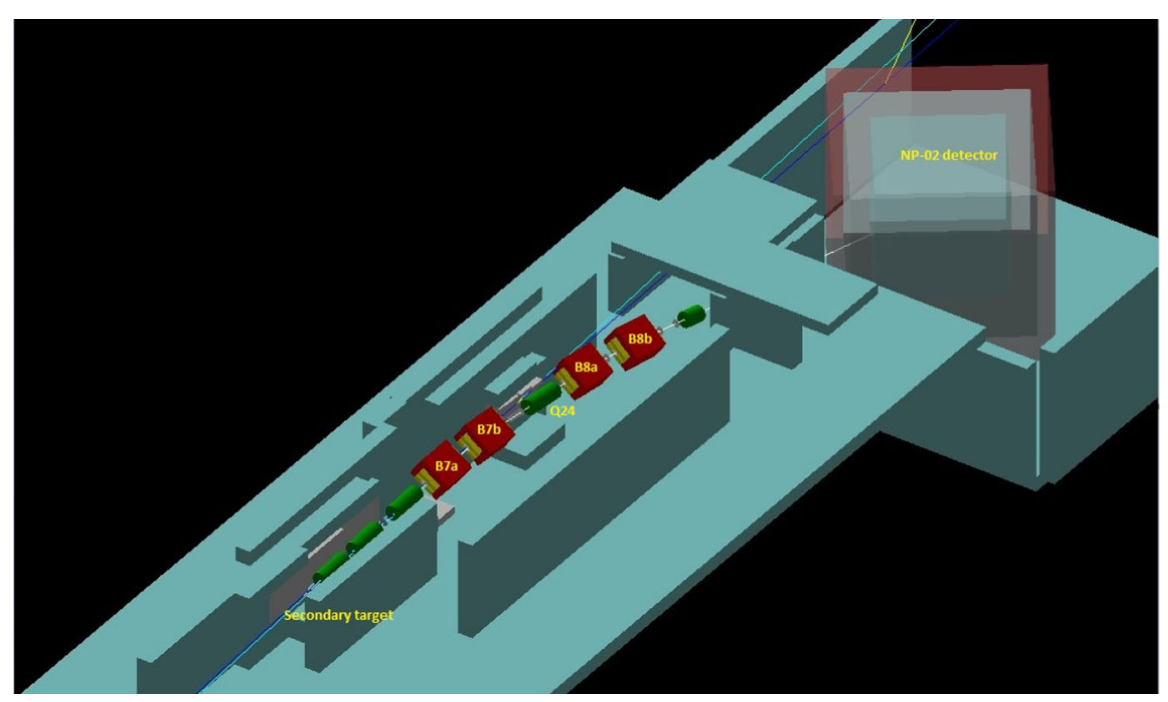

FIG. 10. G4BeamLine ${ }^{\circledR}$ model of the H2-VLE beam line. The green shapes correspond to the quadrupoles, the red ones in the bending dipoles. The secondary target and acceptance quadrupoles are shown inside the shielding bunker and not visible. The dump absorbing the secondary particles is located next to the field-lens quadrupole (Q24). The model of NP-02 detector is shown on the right. (Courtesy: M. Rosenthal and N. Charitonidis)

More specifically, for the VLE electron (or positron) beams the upstream $\mathrm{H} 2$ or $\mathrm{H} 4$ beam lines will be tuned to transport a medium-energy secondary electron beam of 50$60 \mathrm{GeV}$ of moderate intensity of approximately $10^{4}$ particles per pulse. That rate is within reach of the lines, in particular for the $\mathrm{H} 4$ beam that can operate in even higher rates in pure electron mode. As explained above, in this case the secondary target will be comprised of a few radiation lengths of $\mathrm{Pb}$ in the form of a rod of $30-50 \mathrm{~mm}$ in

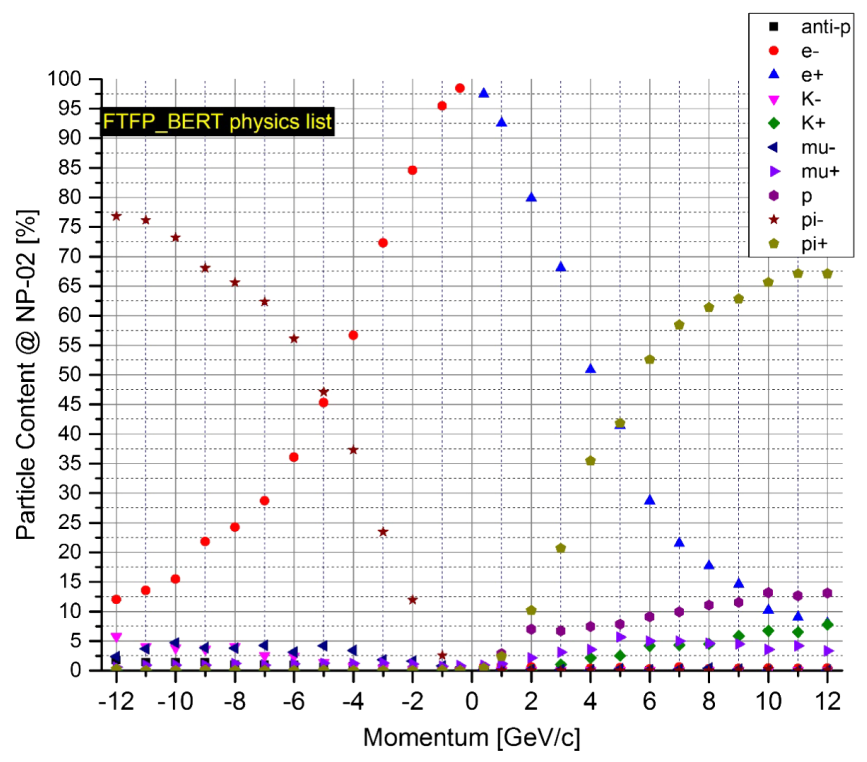

FIG. 11. VLE beam content at the NP-02 experiment, within the beam pipe aperture, for $10^{6}, 80 \mathrm{GeV} / c$ pions impinging on the secondary target. G4BeamLine simulation using the FTFP_BERT physics list. diameter. The resulting tertiary VLE beam will be basically pure, with the only contamination coming from spray of low energy electrons/photons produced in the interaction of the main beam in the apertures of the magnetic elements and other material in the beam line (windows, gas), which can be reduced with the experimental trigger setup and the positive tagging using the installed instrumentation.

Finally, pure low energy muon beams can be produced in the hadron mode by closing the momentum selection collimator. In this case, the last two dipoles in the line will act as a momentum spectrometer to transport the selected beam momenta to the experiment.

TABLE III. Trigger rate at the NP-02 experiment for a W $(0.4-3 \mathrm{GeV} / c)$ target and a $\mathrm{Cu}(4-12 \mathrm{GeV} / c)$ target for the case of H2-VLE. The rates correspond to $10^{6}, 80 \mathrm{GeV} / c$ secondary particles generated at the $\mathrm{T} 2$ target.

\begin{tabular}{lrrrrrr}
\hline \hline $\begin{array}{l}\text { Momentum } \\
{[\mathrm{GeV} / c]}\end{array}$ & \multicolumn{1}{c}{ Electrons } & Kaons & Muons & Protons Pions & $\begin{array}{c}\text { Trigger } \\
\text { rate }[\mathrm{Hz}]\end{array}$ \\
\hline 0.4 & 7 & 0 & 0 & 0 & 0 & 7 \\
1 & 21 & 0 & 0 & 4 & 3 & 28 \\
2 & 17 & 0 & 0 & 7 & 12 & 36 \\
3 & 14 & 1 & 1 & 10 & 30 & 56 \\
3 & 145 & 1 & 1 & 16 & 49 & 213 \\
4 & 117 & 3 & 1 & 16 & 80 & 218 \\
5 & 94 & 5 & 2 & 20 & 100 & 222 \\
6 & 77 & 9 & 2 & 25 & 133 & 247 \\
7 & 69 & 11 & 2 & 28 & 169 & 279 \\
8 & 59 & 16 & 3 & 35 & 193 & 305 \\
9 & 51 & 19 & 3 & 37 & 227 & 337 \\
10 & 46 & 22 & 3 & 45 & 254 & 370 \\
11 & 41 & 27 & 3 & 53 & 268 & 393 \\
12 & 38 & 29 & 3 & 60 & 292 & 422 \\
\hline \hline
\end{tabular}


TABLE IV. Trigger rate at the NP-04 experiment for a W $(0.4-3 \mathrm{GeV} / c)$ target and a $\mathrm{Cu}(4-7 \mathrm{GeV} / c)$ target for the case of H4-VLE. The rates correspond to $10^{6}, 80 \mathrm{GeV} / c$ secondary particles generated at the T2 target.

\begin{tabular}{lcccccc}
\hline \hline $\begin{array}{l}\text { Momentum } \\
{[\mathrm{GeV} / c]}\end{array}$ & Electrons & Kaons & Muons & Protons & $\begin{array}{c}\text { Tions } \\
\text { rate }[\mathrm{Hz}]\end{array}$ \\
\hline 0.4 & 7 & 0 & 0 & 0 & 0 & 8 \\
1 & 19 & 0 & 0 & 4 & 4 & 27 \\
2 & 13 & 0 & 1 & 9 & 15 & 38 \\
3 & 11 & 2 & 1 & 11 & 31 & 56 \\
4 & 92 & 3 & 3 & 17 & 81 & 196 \\
5 & 74 & 6 & 3 & 19 & 99 & 201 \\
6 & 64 & 9 & 3 & 24 & 127 & 227 \\
7 & 52 & 13 & 2 & 27 & 158 & 252 \\
\hline \hline
\end{tabular}

\section{Background at the experiments}

The experiments will be exposed to two types of background: high energy particles (mainly muons) accompanying the secondary beam line and low energy particles produced at the secondary target, along with their interactions with the magnetic elements, the dump and shielding along the line. The particularity of the neutrino platform experiments with respect to background is their large volume, and of being TPC detectors, constantly active with continuous readout. The goal was to minimize the beam-induced background with respect to the irreducible background from cosmic rays reaching few $\mathrm{kHz}$ [14].

For the first type, the VLE beam layout and implementation is such to minimize the exposure of the large detectors to the direct view of the halo of the secondary beam line. As explained above, the detectors are located in deep pits and the VLE beams make large angles in both planes. Assuming that the beam halo of the secondary beam extends to $\sim 1 \mathrm{~m}$ radius around the beam axis, projected onto the detectors, we can see that only the top part of their

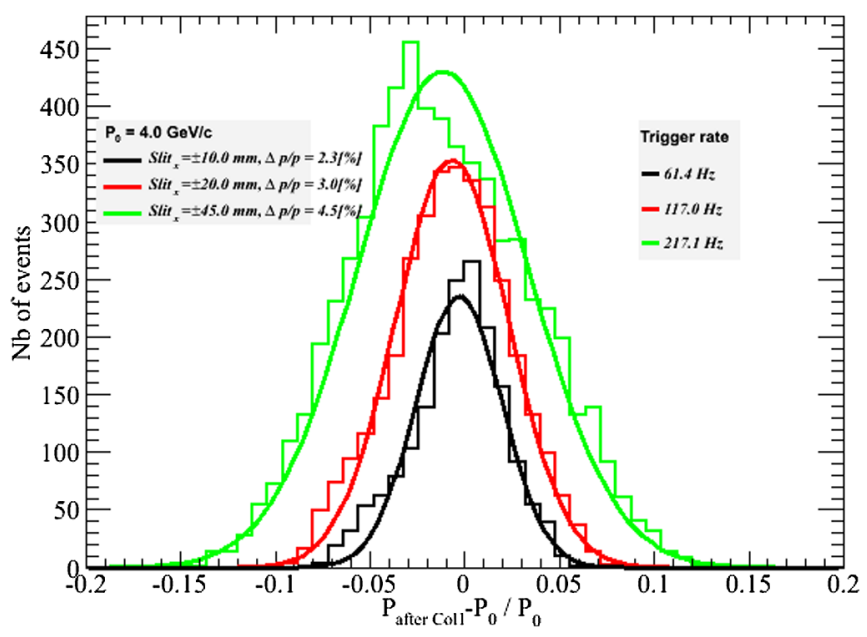

FIG. 12. Momentum bite of the beam for three different collimator slit sizes, for the case of $4 \mathrm{GeV} / c$ beam. The fullacceptance momentum spread is $5 \%$.

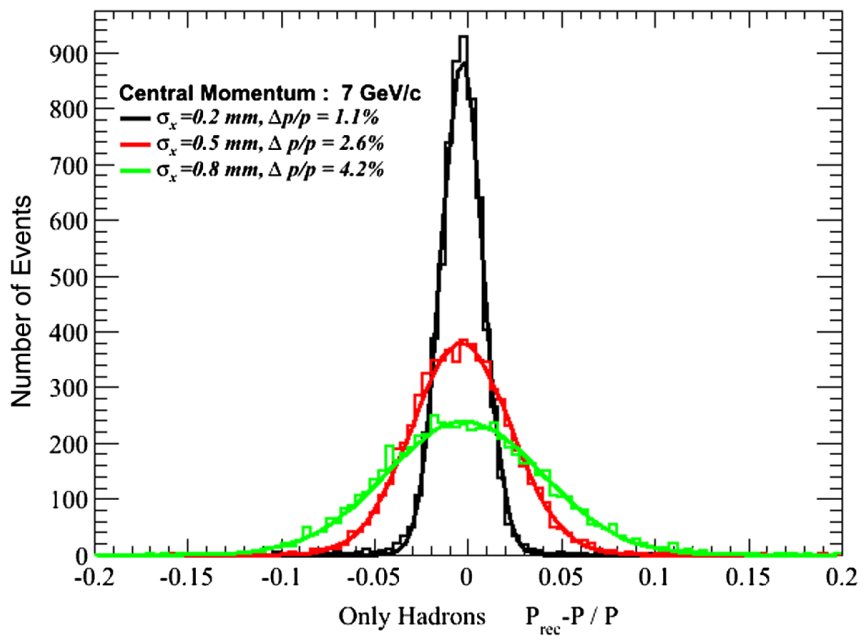

FIG. 13. Reconstructed momentum bite for a $7 \mathrm{GeV} / c$ beam, taking into account the material on the beam line (GEANT4 simulation).

structure and outside the active volume is exposed to this background. If required, during operations the beam halo in the secondary beam can be further reduced by adding side shielding or closing upstream collimators.

The second type of background is somewhat intrinsic to the VLE beam line. At the secondary target a broad spectrum of low energy particles is produced from which only a small fraction will be accepted and transported by the VLE beam line. The rest will be stopped at the magnet apertures, in particular at the first acceptance quadrupoles and the first dipole magnet. The installed shielding from the combination of iron and concrete blocks is very efficient to reduce this background (mainly photons, and neutrons) to acceptable levels for the experiments. What remains is the irreducible background from two sources: particles interacting in the momentum selection collimator (if inserted in the beam line) producing secondaries that can find their way through the downstream bends to the experiment, and in the case of hadron beams from pion decays along the beam line converting the selected pions to muons. These backgrounds can be efficiently handled with the installed beam instrumentation, trigger conditions and particle ID, as experienced in the past implementations (see for example, Ref. [8]).

Extensive simulation studies are ongoing, and on-site measurements during commissioning of the beam lines will be performed to verify the background levels and possibly further optimize the operational conditions of the beam lines.

\section{SUMMARY}

In the framework of the CERN neutrino platform project, two challenging new beam lines able to deliver beams in the $\mathrm{GeV} / c$ range have been designed and are being constructed. In this paper, the key design features of these beam lines and the expected performance has been 
presented. The beam line design was based on extensive simulations using the G4BeamLine tool that provides a handy interface to a full GEANT4 simulation package, broadly used in HEP. The performance of the new low energy beams fully satisfies the requirements for the CERN neutrino platform experiments and constitutes a very important extension of the North Area facility of CERN SPS. The construction of the $\mathrm{H} 2$ and $\mathrm{H} 4$ VLE beam lines is ongoing, and the commissioning is scheduled for early 2018 .

\section{ACKNOWLEDGMENTS}

The authors would like to thank Dr. A. Fabich and Dr. N. Doble for the useful discussions on the initial VLE beam design. Some of the authors would like to also thank Dr. M. Brugger, Dr. L. Gatignon, for the constant follow-up and feedback during implementation, as well as Dr. P. Sala for discussion on issues related to the experiment requests and interface. We thank Dr. Y. Karyotakis and the Laboratoire d'Annecy de Physique des Particules (LAPP) computing department for their support allowing us to run many CPU hours on the local TIER2 cluster and the colleagues S. Girod and V. Clerc for following closely the technical and integration aspects of the new beams, and for providing some of the drawings in this paper.

[1] G. Brianti, SPS North Experimental Area - General Layout, CERN/LAB II/EA/Note 73-4, 1973, https://cds .cern.ch/record/604383/files/cm-p00044885.pdf.

[2] S. Bertolucci et al., Memorandum of Understanding for providing a framework for developing a Neutrino Program at CERN, CERN EDMS \# 1353815v2, https://edms.cern .ch/ui/file/1353815/2/Neutrino_MoU_10-3-14-_complete_ set.pdf.

[3] M. Antonello et al. (ICARUS Collaboration), Precision measurement of the neutrino velocity with the ICARUS detector in the CNGS beam, J. High Energy Phys. 11 (2012) 049.

[4] M. Bishai, E. McCluskey, A. Rubbia, and M. Thomson, Document 10687—v9, Fermilab Document Server (DocServ), Report No. LBNE-doc-10687-v9.

[5] A. Rubbia et al. (DUNE Collaboration), Report No. CERN-SPSC-2014-013.

[6] T. Kutter et al. (DUNE Collaboration), Report No. CERNSPSC-2015-020.

[7] I. Efthymiopoulos and A. Fabich, The very low 1-9 GeV/c tertiary beam extension of the H8 beam line of CERN SPS, arXiv:1206.2184.

[8] S. Abdullin et al. (CMS HCAL/ECAL Collaborations), Report No. CMS NOTE-2008/034.

[9] H. W. Atherton, C. Bovet, N. Doble, G. von Holtey, L. Piemontese, A. Placci, M. Placidi, D. E. Plane, M. Reinharz, and E. Rossa, Report Nos. CERN 80-07, 22-08-1980.

[10] N. Charitonidis, Y. Karyotakis, and I. Efthymiopoulos, Report No. CERN-ACC-NOTE-2016-0052, 2016.

[11] K. L. Brown, D. C. Carey, C. Iselin, and F. Rothacker, Report Nos. CERN 80-04, 18-03-1980.

[12] T. J. Roberts, K. Beard, D. Huang, S. Ahmed, D. M. Kaplan, and L. Spentzouris, G4BeamLine particle tracking in matter dominated beam lines, WEPP120, Proceedings of EPAC08, Genoa, Italy.

[13] N. Charitonidis, Y. Karyotakis, and I. Efthymiopoulos, Report No. CERN-ACC-NOTE-2016-0059, 2016.

[14] C. Patrignani et al. (Particle Data Group), Review of Particle Physics, Chin. Phys. C 40, 100001 (2016). 